\title{
Basic Principles: Some Fundamental Concepts
}

\author{
George A. Pantazopoulos
}

Submitted: 19 February 2015/Published online: 6 March 2015

(c) ASM International 2015

The aim of this editorial is to share a few thoughts concerning the necessary principles which have to be embedded in a "sound and valuable" failure investigation process. Failure analysis targets to root cause analysis and implementation of actions toward improvement. Apart from corporate and business value, which constitutes an essential driving force in a competitive market environment, basic ingredients of a failure analysis and by extension of a research study could be considered as the following:

- Integrity.

- Impartiality/Independence.

- Scientific and Engineering Knowledge/Competence.

\section{Integrity}

It stands as a basic ethical component of human personality which must not be sacrificed or compromised. It is a builtin, standalone virtue in a variety of human actions and processes that contribute thoroughly to the society progress. Many other characteristics are coordinated or are in-line with the term integrity, such as honesty, decency, frankness, modesty, and kindness. Integrity also leads to a unified and constant approach of people facing challenges and difficulties in life. Consistency and professional reliability, with lifelong endurance, require the existence of strong foundations of personality's integrity. Quality and continuous process improvement in international context are originated

G. A. Pantazopoulos ( $\square)$

ELKEME Hellenic Research Centre for Metals S.A., 252 Pireaus Street, 17778 Athens, Greece

e-mail: gpantaz@halcor.vionet.gr from the foundations of individuals' integrity and attempt toward excellence. In-depth and continuous education and true adoption of character's excellence models assist in the development of integrity. A nice term, well known in failure analysis terminology, is "structural integrity" signifying indirectly the absence of (critical) flaws and the presence of uniform/sound structure possessed by a material or component, preventing failures.

\section{Impartiality/Independence}

It constitutes an absolutely necessary approach of a scientist/engineer in the context of a research and failure analysis investigation. The condition of impartiality/ independence strengthens the sharpness of the failure investigation team, in the compilation and interpretation of objective evidence and understanding of the logical chain process which links the events and establishes the causeand-effect relationships in a failure incident. The absence of this basic ingredient ruins the value of the outcome of a failure analysis study. The failure analysis team must be consistent and unaffected against challenges induced by interests of various groups that tend to guide the investigation results to a certain direction. The impartiality and independence of team members together with the release of any emotional restriction or influence constitutes a fundamental ethical rule of practice, which maintains clear and undistorted judgment. This is a necessary condition assisting to consolidate all together the "pieces" of the investigation, in a meaningful and sensible body of knowledge, which drives to inevitable conclusions. The necessary documentation and interpretation of the failure analysis findings shall be infused by the spirit of impartiality and independence. 


\section{Scientific and Engineering Knowledge/Competence}

Deep scientific and technical knowledge ensures the success of the failure analysis study, provided the prior existence of the other two basic components, integrity and impartiality. Multi-faceted scientific knowledge and competence are very frequently required in the interdisciplinary subject of failure analysis and addressed by numerous articles published in Journals and Conferences. Deep, continuous education and competence in Materials Science and Engineering area and expertise in relevant topics, such as Corrosion Engineering and Applied and Computational Mechanics, are significant components of research in the context of Failure Analysis. The involvement of many Engineering disciplines is also unequivocal, mostly in complex failure analysis projects. As a simple example, the evidence of steel shaft fatigue failures that are often originated from corrosion pits on the surface, aggravating stress concentration conditions, suggests the action of combined corrosion fatigue mechanism; the emergence of Mechanics and Corrosion Science field knowledge is required for the interpretation of the results, finding the root cause and implementing corrective actions to minimize the risk of future failures.
The assurance of the above characteristics in failure analysis studies is essential for driving success and it is strongly connected to in-depth and continuous education and selfimprovement, in order to be able to endorse and condense such values to the human personality. Strong excellence models come from enlightened individuals, leaders, scientists/engineers, and philosophers; their teaching results in coalescence and empowerment of a network of ideals and principles which can shape a life guide. Systematic approach is also found in groups and communities, and it is also transmitted from company's strategy and stated vision, mission, and values. Social accountability spreads also the message of society welfare and prosperity, in-line with corporate progress and sustainable development and growth.

Responsibilities arising from leadership positions demand inherent individual's accountability and development of such culture from the close human environment, enabling the creation of a core of ethos and knowledge, e.g., starting from leading our failure analysis project teams or teaching our students/trainees or junior colleagues, offering inspiration models. Our paradigm and mentorship must be strong, bright, enthusiastic, and in-line with the basic principles and beyond, stimulating an auto-catalytic process and contributing to the social cultural growth and development. 